\title{
Trivium
}

Revue franco-allemande de sciences humaines et sociales - Deutsch-französische Zeitschrift für Geistesund Sozialwissenschaften

\section{La science de l'art et la philosophie de la culture et leurs concepts fondamentaux communs II [1919]}

\section{August Schmarsow}

Traducteur : Françoise Joly

\section{(2) OpenEdition}

Journals

Édition électronique

URL : http://journals.openedition.org/trivium/3644

DOI : 10.4000 /trivium.3644

ISSN : 1963-1820

Éditeur

Les éditions de la Maison des sciences de l'Homme

Référence électronique

August Schmarsow, «La science de l'art et la philosophie de la culture et leurs concepts

fondamentaux communs II [1919] », Trivium [En ligne], 6 | 2010, mis en ligne le 05 mai 2010, consulté

le 10 décembre 2020. URL : http://journals.openedition.org/trivium/3644 ; DOI : https://doi.org/

10.4000/trivium.3644

Ce document a été généré automatiquement le 10 décembre 2020.

\section{(c) $(1) \odot$}

Les contenus des la revue Trivium sont mis à disposition selon les termes de la Licence Creative Commons Attribution - Pas d'Utilisation Commerciale - Pas de Modification 4.0 International. 


\title{
La science de l'art et la philosophie de la culture et leurs concepts fondamentaux communs II [1919]
}

\author{
August Schmarsow \\ Traduction : Françoise Joly
}

$\mathrm{Si}$ "l'Antiquité déjà avait reconnu qu'un art donne les choses comme elles sont, et l'autre comme elles semblent être », il faut commencer par dire qu'il ne peut s'agir là que des deux seuls arts plastiques que l'Antiquité considérait déjà comme fondamentalement différents et possédant une pleine autonomie en tant qu'arts particuliers accomplis : la sculpture et la peinture. De plus, c'est un fait généralement établi, avec lequel il nous faut compter, que c'était la sculpture qui, dans l'Antiquité classique, servait de mesure et de critère pour tous les autres arts, qui déterminait toutes les ramifications de la création artistique en les marquant de son empreinte. Et, en l'occurrence, c'est précisément son caractère de "formatrice de corps" [Körperbildnerin] qui était également déterminant pour l'architecture comme pour la peinture. Cette idée est aujourd'hui encore exprimée par ceux qui, sans grande perspicacité, considèrent que dans les temples et leurs colonnes de section circulaire, l'essence plastique serait si complètement et exclusivement présente qu'on ne saurait en réalité parler de configuration spatiale [Raumgestaltung]. C'est ce que pense, par exemple, un spécialiste de l'esthétique comme Theodor Lipps qui, curieusement, ne voit pas que la cella constitue à elle seule un espace intérieur, et qu'un péristyle ouvert, organisé autour du cœur de l'édifice, représente lui aussi une construction spatiale ; sa largeur entre le mur et le périptère, forme toujours de chaque côté une couche d'espace propre que l'on peut donc considérer comme une zone de transition de tous côtés - ne serait-ce que pour le regard qui se porte sur l'édifice -, et elle doit être prise comme telle du fait des intervalles entre les fûts des colonnes. Il est vrai que le rôle de chaque colonne isolée, dans son organisation plastique et la forte autonomie que chacune manifeste même sous le joug de l'entablement qui les réunit entre le sol et le toit, justifie pleinement que l'on parle d'une pensée statuaire et d'une tendance " isolante " dans l'alignement. Cela dit, nous n'avons guère besoin de suivre ce processus dans la 
peinture pour montrer que la conception de la sculpture est également déterminante pour cet art de la même famille, dans les figures des vases peints par exemple ; en effet, à travers la notion de plastique, nous tenons un premier principe fondamental qui s'avère aussitôt commun à la science de l'art et à la philosophie de la culture, pourvu que nous lui donnions son vrai nom: l'individu. Et à travers cette catégorie, nous réunissons les deux domaines, comme nous en avions l'intention.

\section{Sculpture}

2 Poser seul un corps isolé, existant pour soi, indépendant de tout le reste, et donc sans lien avec une architrave transversale ou un «magnifique toit porté par des colonnes ", telle est la tâche de l'art de la statuaire. Dégager l'«individu » humain du flux du devenir et de l'action, l'immortaliser dans sa pleine autonomie et dans sa cohérence, tel est le propos de la sculpture [Plastik] tant qu'elle reste fidèle à sa propre essence et réalise l'exigence supérieure de sa volonté pure. La statue isolée [Standbild ${ }^{1}$ ] est l'image première de "l'individualité de la valeur " que nous comprenons dans le sens d'Emil Lask reprenant lui-même la pensée de Fichte sur la philosophie de l'histoire ${ }^{2}$. Comme nous l'avons dit au début, c'est la valeur de l'existence même que la sculpture incarne dans un matériau durable pour qu'elle demeure aussi immuable que possible, la plaçant ensuite dans l'espace général pour qu'elle s'affirme d'elle-même par ses propres forces sur le théâtre du monde. La figure de l'homme, seule, sur une section découpée et un socle surélevé, dressée au-dessus des fluctuations du monde vivant, en tant que forme pleine, ronde et stable, symbolise la plus originaire des valeurs qui apparaisse à l'homme dans la possession de son propre corps comme d'un corps dans l'espace. Mais c'est en tant qu'elle est image de l'homme, et non pas seulement colonne, que la statue [Bildsäule ${ }^{3}$ véhicule pour les sens et pour l'esprit ce contenu de valeur. Cependant, nous savons tous couramment, surtout pour autant que nous ayons jamais eu affaire à l'Antiquité classique, à l'art primitif en général, qu'un monument dressé en tant que valeur corporelle pure, peut aussi véhiculer le sens d'un état immuable, marquer et souligner en un lieu quelconque le point central de relations complexes et étendues, revendiquer de perdurer en tant que tel et de s'affirmer à travers tout ce qui rayonne en lui et de lui ${ }^{4}$. Nous connaissons le pouvoir du bloc de pierre dressé seul, qu'aucune main humaine n'a encore poli pour lui donner une forme régulière, l'aplanir de tous côtés et le soumettre à la loi de la cristallisation. Cette chose de la nature trouvée telle quelle, à l'état brut, le piquet de bois fiché dans le sol, suffit déjà à servir de support à notre imagination; car il donne déjà l'essentiel, l'axe vertical du corps humain luimême, et sert ainsi de substrat sensoriel, aisément complété par l'apport du sujet qui arrive, de symbole de l'idée de valeur ou d'obscure préfiguration d'une exigence future. Mais sur la pierre taillée, la sculpture "formatrice de corps ${ }^{5}$ » agit de manière purement tectonique, comme sur un membre particulier pour la suite de la construction de l'architecture, pour donner à son œuvre l'empreinte immédiatement reconnaissable de la volonté humaine, pour qu'elle se différencie du fruit du hasard et de butins occasionnels. La première et ultime finalité d'un monument est toujours d'immortaliser une valeur et, dans ce sens, elle est identique à la finalité de toute œuvre d'art. Mais il y a des valeurs qui sont sous-jacentes à l'inconstance de toute vie, qui en tant que facteurs constitutifs dépassent le passage du temps et devraient donc ainsi répondre au désir des hommes de pouvoir lui survivre. Ce à quoi elle vise, c'est dans la plus grande mesure humainement possible, l'immutabilité, et pour y atteindre, 
elle doit exclure, dans la plus grande mesure humainement possible, tout mouvement. Le prix de cette immutabilité, c'est la rigidité, et cette intention de monumentalité passe par la cristallisation de l'existence, du simple être lui-même, au détriment de l'organisation de toute chose vivante (op. cit., p. 170 sqq.). C'est toujours l'abstraction de la chaude plénitude de la créature humaine et des autres créatures apparentées qui en est la condition préalable et qui, dans les formes plus avancées, reste encore longtemps inhérente à toute mise en forme, comme étant la nécessité.

3 À cette sculpture "formatrice de corps » qui pense de manière cristalline et dont la règle est tectonique, nous donnons le nom grec de plastique, qui lui revient de droit, surtout là où elle se propose de donner des formes organiques au noyau stéréométrique de la chose, comme par exemple quand la tête d'homme remplace la sphère sur le poteau planté dans le sol, ou pour le buste sur le socle de pierre de section carrée ; tous deux deviennent alors des "termes $^{6} »$ ou hermès, la combinaison d'un ouvrage plastique avec un support symbolique qui indique en tant que telle la voie qu'empruntera la statue autonome. Conscient de cette étroite relation entre le monument tectonique et la configuration [Gebilde] qu'opère l'art de la statuaire, Alois Riegl a lui aussi parfois parlé d'« individu isolé ", même là où il ne s'agit pas de restituer un homme, mais où l'on se trouve face à un simple bloc de pierre figé, se détachant seulement d'un plan sur tous ses côtés. En insistant sur l'isolement, en qualifiant la construction d'« individu », il lui confère une sorte de personnification ; c'est pourquoi nous faisons bien de le prendre en compte et d'éviter le terme là où il va trop loin ou risque seulement d'induire en erreur, même si nous reconnaissons expressément que son fondement est justifié du point de vue du sens et de l'établissement d'un concept commun.

4 Notre mot allemand de Einzelwesen [littéralement être singulier, séparé, isolé] présente quelque avantage par rapport au terme latin d'« individu » qui nous a été inoculé et s'est donc enraciné dans la science. Ce terme d'origine étrangère d'usage courant comporte en réalité le sens qu'il veut faire passer seulement sous une forme négative, l'individu est quelque chose d'« indivis ", ou d'indivisible; mais il ne rend pas compte de l'essentiel, à savoir de l'être, identifiable ici seulement dans la syllabe finale du substantif [-um] qui le fait apparaître comme un neutre : « ce qui peut vouloir dire un homme et une femme ", ou n'a pas besoin d'être l'un des deux, c'est-à-dire ne porte au fond pas encore de caractère de genre, ou n'entend pas tenir compte de cette spécification de l'être vivant particulier. Einzelwesen donne aussitôt de manière positive l'« individu isolé » comme une unité, et ce singulier est en même temps donné dans une relation avec un concept d'être et de vie, d'apparition extérieure et de vitalité intérieure que nous pouvons résumer par le terme $d^{\prime}$ " activité $»^{7}$. C'est le fait de mettre en avant cette potentialité autonome et isolée qui est essentiel dans la définition que nous devons retenir ici, car elle a déterminé à elle seule toute l'Antiquité pour autant que cette dernière ait été une entité unique. L'individu n'est pensable que comme unité numérique. Et c'est seulement la dissociation de toute relation avec les événements naturels - de la relation moléculaire avec le reste de la nature et de la relation psychophysique avec les organismes de même espèce, de la relation sociale enfin avec les autres membres de la tribu ou de la lignée, de la communauté proche ou de la société plus vaste -, cette abstraction de tous les liens et relations à l'œuvre dans la réalité qui à son tour libère la "valeur d'existence " pour la conscience individuelle. Il faut poser cette considération et la pénétrer de sentiment vivant pour clairement saisir 
qu'il s'agit d'une "idée de valeur» et non pas d'un objet de valeur trouvé dans la réalité, repris tout fait, que l'art humain n'aurait qu'à copier tout simplement d'après la bonne vieille théorie de l'imitation, à répéter ou à reproduire exactement d'après le modèle. Dans la réalité, il n'y a pas du tout d'«individu isolé », et pour l'individu [Einzelwesen] existant, l'essentiel, ce n'est pas son indivisibilité, mais bien plutôt son unité -ou disons tout de suite : son sentiment d'unité, pour ne pas parler avant l'heure de la conscience de soi qu'il s'est constituée ou d'une conscience du moi acquise petit à petit. -L'idée de valeur a aussi quant à elle comme seul repère le corps propre [Leib], d'abord en tant que corps dans l'espace [Körper ${ }^{8}$ ], et elle fait de ce susbstrat sensible le porteur, c'est-à-dire le symbole de son contenu plus étendu, toujours plus large et changeant. La corporéité délimitée, l'unité matérielle qui se manifeste par le toucher et la vision superficiels comme étant close et cohérente, est aussi le fondement de l'art de la statuaire, de l'art plastique que nous désignerons dans son acception étroite comme étant «formatrice d'homme» [Menschenbildnerin]. Mais l'idée de valeur qui suscite et détermine comme motif intrinsèque toute son activité créatrice va bien au-delà de ce fondement donné d'un organisme individuel en faisant abstraction de son lien avec la nature et avec le milieu que constituent les autres créatures, elle exalte l'idée de l'existence constante par opposition à la vie qui s'échappe; et ainsi nous pouvons rapidement conclure que nous avons, ici aussi, affaire à une image mentale de l'ordre de l'« idéal-type » qui prend en même temps pour l'art un sens normatif en tant que finalité et critère de sa réussite. Cette idée de valeur qui exalte tout en simplifiant, l'art ne peut la saisir dans sa pureté que par épuration de la volonté, il ne peut la pénétrer du sentiment de valeur que par la force de l'intensité, comme le commande le prolongement de l'imagination pour passer de l'acte libre de la reproduction au difficile travail de l'acte créateur. C'est pourquoi nous avons toujours dit: "L'âme de la sculpture, c'est le sentiment de soi ! »

5 C'est avec une extraordinaire acuité dans le concept et une abstraction indéniable que la sculpture égyptienne appréhende la valeur de l'existence, avec la volonté de la rendre éternelle. Comme un rapace fond sur sa proie, aussi sûr de son but, aussi imperturbable malgré les obstacles et les distractions, l'esprit, dans sa prise de conscience, se jette sur ce bien qui n'est pas autre chose que le noyau mis à nu de la vie qu'il a reçu par sa naissance et qui, ainsi, lui appartenait donc déjà par nature. Dans le matériau le plus rigide, le plus inébranlable, dans sa restitution de l'image de l'homme, elle se limite aux traits essentiels communs, fixe d'abord le type, non pas seulement le type de l'homme en général, mais celui du peuple égyptien, de sa race propre ou du souverain, auquel elle rend hommage. En un patient labeur, sans se laisser rebuter par le temps qu'il y sacrifie, le sculpteur taille les parties du corps sur une pierre très dure qui n'autorise qu'une simplicité schématique. Impitoyable jusqu'au fanatisme, il retient la seule chose essentielle, la permanence de l'existence corporelle, en lui donnant la dimension des colosses. Il faudra attendre les Grecs pour que soit coulé à l'image de l'homme dans le marbre le sentiment de soi, enrichi de la chaleur de la vie même, comme capturée dans la jouissance du bonheur de l'existence, et qu'ainsi le spectateur aussi en soit comblé de bonheur. Mais ce léger souffle de respiration qui s'échappe des lèvres entr'ouvertes, la plénitude renflée des membres ne doivent pas laisser croire que tout signe de fugacité est écarté, toute relation au métabolisme annulée sous l'enveloppe de ces formes fixées dans leur rondeur bien que la jeunesse éternelle de cet inaltérable épanouissement soit une condition réalisée avec autant d'évidence que de réussite. Là aussi une part de libre abstraction dans le sens de l'idée de valeur réalisée, 
là aussi une exaltation de certaines propriétés qu'il s'agit de fixer pleinement, mais en accueillant plus largement la riche diversité des traits de l'existence vivante, dans la beauté des corps harmonieusement formés avec toutes les aspirations pour l'usage dans la compétition de forces également développées. Dans ce cas encore, la sculpture part d'un type commun, mais choisit résolument les âges les plus propices, préfère le sexe masculin, tant que la tâche garde sa rigueur spécifique, authentiquement hellène. Alors le type se développe à travers l'heureuse union avec l'individuel pour devenir un idéal, c'est-à-dire le résultat tout à fait concret de la représentation de la valeur que sont les représentations bien connues des dieux de la Grèce 医 l'idéal objectivement existant réalisé dans l'œuvre d'art et que j'ai défini comme "l'unité supérieure entre l'individu et le type ». Cette conquête ne s'explique de manière satisfaisante, mais très naturellement alors, que par une action de l'idée de valeur dans l'imagination créatrice qui ne vise pas à restituer n'importe quelle chose donnée dans la réalité en se contentant de la copier par reproduction, mais au contraire, dès l'acte de reproduction, assure la liberté de l'esprit tant elle reste liée aux impressions ressenties et aux expériences vécues. Elle épure et idéalise, elle simplifie et intensifie une image originaire de la valeur qu'elle se propose de fixer pour l'éternité : la beauté du corps [Leibesschönheit] dont procède la découverte par l'homme de toute beauté. Et à travers le nombre croissant des œuvres de l'individuel en tant qu'il est une forme unique, née et issue de conditions limitées dans le temps et le lieu, on en arrive à l'ultime forme d'expression de cette idée de valeur que la sculpture "formatrice de corps " puisse réussir à produire en gardant intacte la cohérence de son essence - l'immortalisation de l'individu historique, du contemporain, le portrait d'une personne particulière. Mais cette immortalisation s'accomplit aussi toujours chez les Hellènes sub specie quadam aeterni, c'est-à-dire dans la transposition aux "régions supérieures où résident les formes pures ", là où les scories des événements terrestres sont tombés dans le feu de l'enthousiasme.

6 Après avoir ainsi fixé le premier principe fondamental pour l'art de la sculpture, et donc pour toute la culture de l'Antiquité, nous pourrions poursuivre la confrontation de l'homme avec le monde dans lequel il est né à partir des observations biologiques et psychologiques de la recherche la plus récente ${ }^{9}$. L'individu humain est, selon l'idée de valeur qui se forme en lui-même, un tout unique même si, en tant que créature organique qui naît et meurt, il ne peut pas être un individu isolé comme l'idéal de valeur que propose son art plastique. Ainsi le corps est-il toujours là avant l'âme, et le sentiment du moi est antérieur à la conscience de soi dans sa totalité articulée. Mais plus la vie avance, plus l'activité psychique, en même temps que ses intérêts vitaux supérieurs, va au-delà des simples fonctions corporelles. L'homme donc, ne devient un individu intellectuel ou psychique que lorsque se déclenchent en lui des forces dont l'action est liée à des expériences conscientes ${ }^{10}$. Ce changement, nous l'observons sur le terrain de la statuaire à partir de l'Antiquité tardive dans l'évolution de la culture chrétienne au fil des siècles. Naturellement, une différence tout à fait primaire entre les hommes réside dans les objets qui influent sur leur comportement possible et déclenchent de ce fait les "affects sensibles » [sinnliche Gefühle] qui sont les racines de la création artistique, et cette différence de prédispositions initiales, dans les instincts d'une race par exemple, est déjà une différence de valeur d'une portée fondamentale. Là aussi, il faut revenir au corps inné, légué par les ancêtres et reconnaître encore une fois le bon droit de la sculpture "formatrice de corps». Alors, la confrontation de l'homme [avec le monde] s'accomplirait d'abord à partir de son corps face au reste du 
monde des corps dans l'espace, ou pour la statuaire d'abord face à l'espace complètement indéfini, non inclus dans la présentation, et qui constitue ainsi seulement un pôle négatif. Si nous adoptons la distinction soigneusement opérée par Max Scheler entre les notions de Körper et de Leib, nous aurons à opposer en second lieu à ce concept de Leib (qu'il divise à nouveau en Leibkörper et Körperleib) celui de milieu [Umwelt], qui au sens des biologistes d'aujourd'hui doit être divisé entre le monde de la perception [Merkwelt], réceptif (rezeptorisch) et le monde de l'action [Wirkungswelt], efficient (effektorisch). - Il en découle la confrontation du Moi avec le monde extérieur. La notion de "Moi» peut, dans ce raisonnement, être en relation à travers les expressions de « corps-moi » [Leib-Ich] et de " corps-âme » [Leib-Seele] d'une part avec le domaine de symptômes précédents, d'autre part avec le domaine de symptômes suivants (la psychè), mais s'avérer sinon être le centre du monde intérieur. - Nous en arriverions enfin à l'opposition entre la personne d'une part, dont, du fait de la division courante entre âme et esprit, nous devons clairement dégager le concept dès qu'il s'agit de reconnaître un individu non pas au sens d'être vivant organique, mais aussi d'être vivant psychique, et d'autre part le monde concret de cette personne qui peut être appelé ici «monde en général » parce qu'il est « un être individuel, partout concret et étant absolument, et non pas une simple idée au sens de Kant ». Ici s'ouvrent toutes les perspectives de la personnalité intellectuelle et de son univers affectif et idéel.

7 L'individu humain, qui ne reste libre que dans l'idée de valeur de l'art statuaire, est affecté dans la vie par une innombrable quantité d'influences diverses; il entre dans des relations d'échange infiniment complexes qui, du fait de sa sensibilité pour telle ou telle action extérieure, le déterminent à leur tour selon sa spécificité individuelle, cette dernière réagissant en retour pareillement sur son comportement vis-à-vis de son milieu propre, le monde de la perception comme le monde de l'action. C'est seulement dans cette interaction que se révèle la différence entre sa fonction somatique et sa fonction psychique comme un véritable pouvoir. Mais ce n'est que dans l'ensemble des relations sociales avec les autres hommes et dans l'échange réciproque avec eux que l'individu [der Einzelne] devient le sujet intellectuellement agissant et capable d'agir auquel nous pensons selon ses deux aspects, le corporel et le psychique, quand nous le qualifions d'individu ${ }^{11}$. La statuaire qui entreprend dans la forme humaine, donc dans, avec et sous l'individualité des sens, d'exprimer également la personnalité morale, c'est-à-dire finalement de transmettre la teneur intrinsèque que Fichte a appelée l'« individualité idéale ", cette statuaire cependant doit renoncer à la montrer dans ses relations avec le monde; car elle ne représente que l'individu [Einzelwesen], seul et pour soi, en n'incluant plus du tout dans sa présentation l'espace qui l'entoure. Ce sont les plus grands miracles de l'art du portrait en sculpture, non seulement dans l'Antiquité, mais aussi dans les temps modernes où l'individu historique apparaît dans sa valeur d'unicité singulière et où l'amour de l'artiste cherche toujours à immortaliser cette valeur d'individualité comme valeur d'existence ${ }^{12}$.

\section{Mimique}

Mais longtemps avant de pouvoir réaliser cette mission de suprême concentration dans une unité close et autonome, la vie même dans son procès invite à élargir à deux ou plusieurs corps l'un à côté de l'autre, que ce soit pour former un groupe, ou aussi, à l'aide du fondement du bloc de pierre, de la plaque de bois ou de métal, pour former un 
relief. Mais dans les deux cas, ce n'est plus la forme et la position du corps seules qui réalisent la valeur, mais le mouvement du corps et ainsi le geste expressif qui porte la valeur des relations et donc véhicule une valeur nouvelle, d'une autre sorte. Un couple d'amis ou de compagnons de lutte, un couple d'amants, également, dans une formation stable, les proches autour du membre défunt d'une famille tels qu'ils sont réunis sur une stèle mortuaire, constituent des exemples de cette sorte d'élargissement aux valeurs de la vie; et la mise en regard de deux individus qui va susciter la manifestation de leur spécificité et de leurs différences, ouvre un vaste champ de possibilités, jusqu'au sommet que représente l'antagonisme ${ }^{13}$. Mais dans ce domaine, un autre art a depuis longtemps anticipé sur le travail de la sculpture. Oui, nous n'aurions en fait pas eu besoin d'élargir autant les prétentions de la sculpture, qui en réalité dépasse les limites de son essence originaire. Car le bon droit de la mimique est et reste d'apparaître à sa place originaire et de s'affirmer là où elle est par nature. Il est indéniable que c'étaient des valeurs mimiques qui, dans le groupe et dans le relief, venaient accompagner les valeurs corporelles pures, et plus les motifs se font vivants, plus ils refoulent nécessairement le propos essentiel de la sculpture en menaçant forcément la valeur de l'état stable par leur attraction pour l'imagination. J'ai toujours insisté sur le fait que nous ne pouvons pas traiter les arts plastiques isolément, si nous ne voulons pas perdre la clé de l'ensemble du royaume de l'art; nous devons inclure toute la série des arts musicaux et littéraires [musisch, pour les trois Muses originaires de la musique et de la littérature] ou plutôt des arts de la « forme de vision dans le temps " parce qu'il ne doit en manquer aucun si l'on veut appréhender leur noyau commun et la relation qui les traverse.

9 Seulement, on méconnaît encore trop souvent le fait que l'ensemble que je comprends ici sous le nom de mimique constitue un domaine artistique tout à fait propre et initialement autonome dont le principe de mise en forme créatrice ne doit pas se voir spolier de son droit d'aînesse sous prétexte que le sort a voulu qu'il s'égare en devenant un allié très convoité dans toute une série d'autres arts. Le désir de voir l'autonomie d'un art se présenter tout au long d'une existence historique, d'en trouver encore aujourd'hui des manifestations et des séries ininterrompues d'irréfutables témoignages repose sur un préjugé, celui de ne pas admettre l'office que remplit la théorie qui consiste à rechercher des traces et combler les lacunes d'une tradition quand elles interrompent la continuité du nécessaire état des faits, ou bien quand cette tradition a échoué, quand le passé a disparu sans histoire parce que cela se trouvait déjà donné par la nature même de la chose et dans l'absence de moyens (ici graphiques) permettant de la maintenir éternellement. La mimique jaillit directement de la réalité de la vie et replonge aussi dans le courant de ces forces supérieures qui emportent tout sur leur passage ; mais la recherche historique peut encore parvenir à ressusciter le Dieu brisé à partir des membres épars d'Osiris, à la seule condition que la science de l'art sache reconnaître le principe de vie quel que soit le lieu où il a exercé et exerce encore sa capacité à engendrer quelque chose. La question est la même ici que pour tout autre art particulier, c'est celle de l'idée de valeur à laquelle cet art doit son pouvoir de création - quelle est la valeur particulière qu'il veut représenter et véhiculer ? Ce qui implique aussi d'établir pour la mimique en tant qu'art un «idéal-type » qui soit son principe fondamental non seulement pour une période historique donnée, mais qui ait aussi une validité générale et garde, au moins dans ses caractéristiques principales, un pouvoir normatif pour tout ce qu'il produit, quelques multiples et diverses que soient les variations à travers lesquelles nous l'accompagnons au cours de son destin. 
10 Toute valeur qu'il nous faut reconnaître dans son droit originaire et imprescriptible doit être donnée sous une forme non seulement visible, mais aussi perceptible par les sens et ainsi accessible au sentiment humain. Mais là où, comme dans ce cas, elle est elle-même coulée dans la vraie vie, il faut de plus un moyen unique pour l'arracher à la réalité du quotidien et la préserver de toute confusion avec lui. De même que dans la sculpture la simple valeur corporelle, pour accéder à l'éternité, doit se distinguer de la valeur donnée dans la nature par le choix d'un matériau rigide froid, et que toute tentative insensée de vouloir tromper les sens en se rapprochant de la couleur et de la matière du corps humain vivant (cabinet de cire) récolte aussitôt désapprobation et opposition générales - de même, ici, ce sont les mouvements du corps et la gestuelle en tant que valeur que saisit la mimique, c'est-à-dire en tant que valeurs d'expression, qui sont au premier plan, mais en évitant rigoureusement tout mélange avec d'autres moyens d'expression comme le son de la voix, la sonorité de la langue. Seule la mimique absolument muette, sans aucun accompagnement musical, est le libre jeu qui peut se développer jusqu'à devenir un art dont nous pouvons dégager la pureté et qu'il faut reconnaître comme un art indépendant. Les limites à l'intérieur desquelles l'homme vivant est lui-même le support de la forme d'art sont étroites. Au-delà, nous en venons aussitôt à des liens entre deux arts, et ainsi à des arts doubles, aux royaumes intermédiaires de la transition; d'un côté, l'alliance avec la musique entraîne infailliblement le mouvement des corps et l'expression en rythme, la danse donc, et de l'autre côté, l'alliance avec la langue, l'élément de la poésie donne naissance à l'art du théâtre [Schauspiel] ${ }^{14}$ qui soutient le poète dans la présentation dramatique; et la double alliance avec la musique et la langue dans le chant fait même surgir un genre mixte encore plus complexe avec différents principes artistiques dont la théorie ne peut rendre compte que si elle dénoue ces liens complexes et s'attache à soigneusement distinguer ses résultats pour étudier d'abord la synthèse de chacun de ces couples pour soi.

11 L'art mixte de l'acteur [Schauspieler] a même emporté avec lui le nom de la mimique pure ; car cette dernière est elle-même un « jeu de vision » [Schau-spiel] au sens strict, un jeu que l'on doit juste voir [schauen] et non pas en même temps entendre dans celui de l'acteur, et c'est un authentique «jeu » [Spiel] qui ne veut pas se faire sérieux et ne doit pas se confondre avec la réalité - même si le contenu, devenu un art, qu'il entreprend de représenter et véhiculer est sérieux et pleinement porteur de valeur. Neutraliser la langue, exclure les sons, longtemps même les expressions du visage que recouvrait le masque - telle était la règle de ce jeu. Cette règle supprime des éléments tellement importants de la vie réelle que son jeu muet peut prendre librement son envol et quitter le terrain du quotidien pour les contrées de l'imagination, afin de retourner comme un gant les valeurs de l'univers intérieur et les proposer à l'intuition sensible, pour exalter le pur univers de l'expression et en faire une expérience vécue des autres hommes. Son silence justement est le pouvoir magique qui lui donne des ailes $^{15}$.

12 Partout où un individu humain en rencontre un autre, un besoin intérieur d'échange se fait sentir comme moyen naturel de compréhension pour tous les êtres vivants dans le commerce qu'ils entretiennent. Avant même encore que la voix ne se manifeste ou que la langue ne vienne à son secours, l'œil saisit déjà le sens du mouvement du corps, de la position et du geste. C'est là-dessus que la mimique se construit, exclusivement comme un art du geste. Et c'est ainsi qu'elle commence à agir ; de la même manière que nous 
passons de l'individu [Einzelwesen] dans sa singularité au duel [Zweizahl] ou au pluriel [Mehrzahl], nous ajoutons au premier principe fondamental de l'individu isolé celui qui suit directement dans sa communauté. Elle est aussi économe de figures que le groupe statuaire ou le pur relief; car elle sait que la moindre distraction et la plus infime dispersion de l'intérêt compromettent l'unité de relations qui, de toutes façons, ne peuvent s'accomplir que dans un mouvement de pendule d'un individu à l'autre. C'est bien par la succession dans le temps des mouvements expressifs que la mimique se distingue de la sculpture "formatrice de corps ", et c'est l'attitude de l'homme vivant, dans sa mobilité et son inconstance propres, qui détermine la technique de l'art de la gestuelle muette. La technique mettant en jeu une combinaison de toutes les parties du corps vouées à d'amples mouvements réclame qu'on ne perde jamais de vue l'unité corporelle à tout moment de la succession précipitée [des gestes] qui s'accélère toujours davantage. D'où résulte donc, quand sont associées deux de ces unités (deux mimes), que l'on passe sans arrêt de l'un à l'autre. Parfois seulement un rapprochement des deux protagonistes intervient au moment essentiel, pour marquer leur accord ou bien la contradiction. S'il y a une troisième personne, elle ne peut que se joindre à l'un ou à l'autre des deux mimes pour être son partenaire - égal ou subordonné - ou bien se dresser comme dominant les deux autres ; car sinon, dans le cercle étroit des figures, sur le podium au milieu des spectateurs, la communauté de vision se déferait, et la continuité de ce qui porte toute l'unité de l'œuvre d'art mimique serait interrompue par un vide dans le déroulement rythmique de l'action. Tout arrêt dans l'activité et dans la gestuelle des joueurs suffit à concéder à l'intuition des corps, qui relève de la sculpture, un poids trop grand par rapport à l'intuition des mouvements, qui relève du mime, et c'est ainsi que se produit comme un vide dans le déroulement de la représentation de ses valeurs d'expression fugitives. Le caractère transitoire des mouvements du corps et la rapidité de leur enchaînement - même si le jeu des physionomies est encore annulé par la présence du masque - comportent un autre trait essentiel de la mimique pure comme art autonome; justement le trait essentiel pour son principe de mise en forme le plus spécifique, à savoir le caractère typique de ses moyens de représentation. Ce n'est plus l'individu qui domine ici, c'est le type ; c'est pourquoi, à propos de ce spectacle, nous parlons tout naturellement de personnes ou même de figures. Ce n'est pas la totalité des qualités particulières d'un individu qui peut être mise en valeur, mais seulement ce qui fait partie des propriétés constantes de la nature humaine commune, ou bien, pour reprendre les termes de Duns Scot, le si subtil penseur du Moyen Âge, ce n'est pas ici la haecceitas, mais la quidditas qui entre en jeu. Seules sont présentées les variations les plus simples, directement compréhensibles, et les oppositions les plus évidentes d'âges, de sexes, de races ou de tempéraments, seules les différences ordinaires manifestes même pour quiconque n'en serait pas familier, et non pas les nuances intimes et les motifs entrelacés que l'observateur proche, par exemple, sera le seul à savoir déchiffrer, et encore, seulement après une longue fréquentation. - Dans la mesure où, de toutes façons déjà, la langue manque, où le timbre de la voix ne joue pas, le secours de tous les autres motifs intérieurs que seule la voix peut faire passer fait défaut, de même que fait aussi défaut tout l'ensemble de relations motivées que le poète nous révèle dans la poésie. Ici, dans ce théâtre sans paroles, seule la visibilité extérieure agit - pas d'oreille tendue dans le silence d'un cabinet, pas de leçons à tirer de séries cachées d'idées ou de débordements affectifs liés à des représentations imaginaires. C'est le typique qui reste la condition de toute communication [Verständigung]. 
13 Et comme toute culture repose sur ces outils de communication quand elle veut aller au-delà de la nature humaine, il s'ensuit un nouveau point de vue pour la philosophie de l'histoire: sans type, pas de tradition, car pas de communication, pas d'expression saisissable de nos mouvements corporels, cette expression ne surgissant que de la confrontation avec le milieu commun. Seul le fondement typique de toute gestuelle, de toute attitude, de tout «signe » des sens pour des émotions psychiques, donne la clé de leur compréhension. Toute note par trop particulière d'un individu rend plus difficile leur transmission, sinon rapide comme l'éclair, à un autre individu. D'ailleurs, de la même manière, dans un pays étranger, longtemps, nous ne comprenons que ce qui est typique.

14 C'est pourquoi toute la pantomime muette ne peut que constituer ses moyens d'expression en un système de valeurs expressives typiques et compléter ce système par l'invention. Cette «symbolique " prend valeur de convention dans son usage plus large de sorte que nous sommes en droit de la qualifier de «langage gestuel», mais ce n'est pas tout de suite le cas. Seuls des motifs typiques, et avec eux aussi des personnes typiques, peuvent ici être porteurs de valeurs; eux seuls sont à la fois possibles et nécessaires. C'est grâce à ces motifs que le pétulant méridional, familier de cette pratique, pourra conduire des dialogues entiers et aligner des performances cohérentes où la mimique muette réussit à elle seule à captiver l'imagination des spectateurs en les tenant en haleine longtemps. Nous sommes là aux confins de la poésie.

Les valeurs mimiques sont des valeurs typiques qui procèdent de la communauté des hommes en tant qu'êtres sociaux; les concepts fondamentaux relevant de la philosophie de la culture que manient l'ethno-psychologue ou le sociologue peuvent directement s'appliquer à ce champ de la vie sociale du point de vue la science de l'art ; celle-ci refusant encore curieusement de se convaincre que, pour donner le contenu de tout relief, de tout groupe plastique, voire même du motif d'une statue, elle ne peut se passer du secours de la mimique qu'elle met en toute quiétude sur le compte du sculpteur ou du peintre au lieu de s'interroger plus avant sur l'origine artistique de ces moyens. Pourtant, elle devrait entre-temps saisir elle aussi le caractère indispensable du principe absolument autonome et hautement spécifique de cette mise en forme expressive, dans le champ des autres arts de toutes façons. J'ai toujours souligné la part centrale que prend le mouvement expressif dans le mouvement vers la finalité dès que l'on étudie l'activité de nos organes du toucher et des outils naturels de toute production à partir d'une matière plastique. Et quand l'on considère la sculpture monumentale du Moyen Âge chrétien, on est bien obligé de souligner qu'au lieu des valeurs plastiques pures, elle préfère les valeurs mimiques, qu'elle ne présente donc plus ni la corporéité de la ronde bosse sur laquelle repose presque exclusivement la sculpture antique, ni la forme organique de corps réguliers, mais bien les mouvements $\mathrm{du}$ corps et les gestes, des valeurs expressives portées par des œuvres qui se rapprochent souvent de la forme stéréométrique d'éléments tectoniques de construction. Les jubés d'église, les voussures des portails et les étagements de la façade constituent une démonstration des relations typiques qu'entretiennent les personnages bibliques, que ce soit celles d'un couple, ou d'une série entière, comme les relations de Marie et Gabriel dans l'Annonciation, des deux femmes dans la Visitation, de deux prophètes et apôtres, ou des séries entières de rois ou de représentants de l'Ancien ou du Nouveau testament. La statue isolée, dans son tabernacle au sommet d'un pilier ou d'un tympan, est elle aussi dans une relation mimique avec tout le reste du système de 
la doctrine de l'Église que l'ensemble des œuvres d'une église doit proclamer. Ainsi, ces sculptures ne sont-elles pas seulement un témoignage de l'intériorisation chrétienne dans le sentiment de valeur pour l'individualité, elles inscrivent également l'individu dans un ensemble de relations plus large, donc dans une "totalité de valeurs » qui ne peut apparaitre que dans la communauté. En revanche, elles ne franchissent pas les limites des valeurs expressives typiques qui sont maintenues ne serait-ce que parce que l'égalité de toutes les âmes devant le Très Haut, la fraternité de tous les enfants du Père éternel, l'intégration toujours réitérée de l'individu à sa place dans le royaume de Dieu, sur la terre comme au ciel, est la condition générale de toute individualité de valeur et reste donnée avec la seule attitude de valeur du monde intérieur. En même temps que le sentiment de soi de l'Antiquité s'efface au profit d'un retrait sur la conscience de soi de la personne, on voit aussi se défaire l'« individualisme » naïf. Déjà le rationalisme de l'Empire romain affirmant l'indépendance de l'individu isolé par rapport à toutes les relations sociales et historiques, permet à bien des égards une soumission presque étouffante de l'individu à la généralité de la valeur. Le christianisme, en revanche, défend un individualisme qui, dans la valeur de l'âme singulière, reconnaît l'affirmation autonome de l'individualité de la valeur par rapport à toutes les valeurs simplement abstraites, mais exige, à travers l'idée de la communauté fraternelle, que le singulier se range dans une totalité de valeur plus vaste (cf. Lask, op. cit.,. p. 16 et suiv.).

Les concepts fondamentaux des sciences sociales et de la philosophie de la culture qui, à travers la mimique, entrent déjà dans la science de l'art, passent donc, aussitôt après l'individu, à son dédoublement et, à partir du couple, à tous les pluriels encore perceptibles en tant qu'unités jusqu'à une multiplicité où les relations mimiques ne fonctionnent plus, où l'on ne peut donc avancer jusqu'aux riches relations d'un système global qu'à travers la seule symbolique conventionnelle, c'est-à-dire vers une unité qui ne s'accomplit plus qu'intellectuellement comme résultat d'un travail d'imagination, d'un acte de pensée. On touche là encore une fois aux frontières de la poésie.

Mais en s'attardant encore un instant sur l'œuvre d'art totale qu'est la cathédrale gothique, nous verrons alors s'éclairer une autre idée qui ne devrait pas échapper à la science de l'art. La distribution des statues dans les articulations de l'architecture répond au principe d'une mise en place en alignement soit vertical soit horizontal, ou alors en groupement devant un arrière-plan effectivement donné ou spatialement suggéré, de sorte que la vue d'ensemble se rapproche plus ou moins de la conception du relief. Vient s'y ajouter le véritable art du relief, avec son contenu narratif ou dogmatique, qui vient compléter la fonction des figures individuelles à l'intérieur de la communauté, ou montrer les figures des saints dans leur échange mutuel vivant, dans leur relation avec leurs protégés sur la terre, dans le douloureux combat avec leurs adversaires. Nous touchons là par tous les bouts aux limites de la forme de vision mimique pour aborder indéniablement le royaume de la conception picturale et de ses formes de représentation, que ce soit dans le cadre clos du tableau, ou dans le déroulement de fresques murales, ou enfin dans la peinture de tout un espace intérieur qui constitue, par sa forme architecturale, un système articulé. Mais, là aussi, nous pourrions directement trouver le passage de la mimique à la peinture dans le stade tardif de l'évolution de la mimique, quand elle remplace le podium librement placé au milieu de la foule des spectateurs contre une scène en largeur, mais encore sans profondeur devant une cloison, sur une place en plein air ou dans un espace intérieur 
sous un toit. Là aussi s'accomplit la transition entre la vision en trois dimensions de la statuaire et la vision en relief depuis le devant.

\section{Peinture}

18 Ce que la mimique dans son contenu si divers prépare et a exploité dans des relations toujours variables se voit repris, selon le même processus que pour la sculpture dans le groupe et le relief, par la peinture dont l'idée de valeur dominante est, comme nous l'avons définie, la présentation de l'ensemble des relations visibles entre les personnes et la scène sur laquelle elles sont montrées, ou entre les choses et le monde. Comme la sculpture dans les groupes de statues ou dans le relief encore, elle fixe d'abord toutes les relations qui ne se peuvent comprendre que dans l'ordre du visible, par le truchement de l'œil. Et le tableau, « unité supérieure entre les corps et l'espace », est la conquête qu'elle propose comme sa valeur la plus spécifique en tant qu'art indépendant ${ }^{16}$.

19 S'il faut maintenant non plus s'en tenir aux points de vue de la science de l'art ou même de l'observation formelle des styles, mais élargir cette fois volontairement le regard à la philosophie de la culture, alors apparaît en ce qui concerne la peinture une surprenante communauté de concepts fondamentaux qui nous invite à réévaluer d'une manière plus positive que cela n'avait été le cas récemment tout ce qu'avait pu apporter la bonne vieille esthétique idéaliste.

La théorie générale des principes de l'art plastique arrive aujourd'hui très vite à un point où l'on revendique que les arts particuliers fassent l'objet de phénoménologies particulières, quand on ne va pas jusqu'à remettre en cause le maintien du contact. D'un autre côté, si l'on tente de déduire des « concepts fondamentaux de l'histoire de l'art » d'utilité générale uniquement à partir de périodes particulières bien définies et précisément délimitées de l'évolution des styles, cela débouche sur un malentendu sensible, à savoir que l'on établit et exploite ainsi "des catégories artistiques » sans égard pour les champs artistiques et pour les exigences spécifiques que l'effet des œuvres d'art elles-mêmes pose à la forme de représentation, ce qui, dans la culture de chaque époque, constitue leurs prémisses. Les chapitres complémentaires ajoutés parfois sous le titre "Considérations par motifs " témoignent seulement du péché d'omission commis dans l'organisation générale [du livre, de la théorie], à savoir le fait d'avoir négligé les points de vue de l'histoire de la culture en général ou les contenus de la représentation. Or nous nous entendons sans aucun doute pour reconnaître le progrès qu'a signifié pour la science de l'art le fait de «distinguer les formes fondamentales typiques de la percée artistico-représentative dans le monde de l'intuition à travers une certaine structure de l'expérience esthétique de la valeur ${ }^{17}$ ", et les faire valoir dans leurs droits ; mais cela se voit sanctionné quand on ne tient plus compte «des objets que déterminent l'éthique et l'intuition de monde respectives des autorités, que l'art doit exalter. » Quand nous sentons qu'il faut « faire de nouveau la distinction entre ces formes typiques et les lois esthétiques et techniques consciemment appliquées ", il ne faudrait justement pas oublier qu'elles sont aussi liées à une tradition inconsciente léguée de génération en génération et dépendent d'habitudes des états culturels qui ne se modifient que lentement. Il n'est pas possible d'admettre les procédés purement artistiques de la critique de style, appliqués par exemple à la Renaissance et au baroque, sans faire la différence entre les œuvres, les 
tableaux qui relèvent à l'origine de l'art religieux et ceux qui doivent leur existence au libre choix d'un cercle purement séculier, cultivé, au seul goût esthétique. Il est parfois même tout simplement absurde de transposer arbitrairement les critères de l'architecture solennelle et de la claire rigueur formelle évidents pour le retable d'un lieu de culte, à l'ornementation d'appartements princiers relevant d'une représentation mythologique profane quelconque ou de compromettantes aventures amoureuses; ou bien, inversement, de comparer directement la liberté conquise d'un côté dans la facture picturale, dans les charmes du coloris, dans le clair-obscur évanescent, comme par exemple dans les Danaé ou Io du Corrège, avec la construction et le langage formel d'un tableau d'église de la même époque. Cela pourrait donner un jeu d'antithèses à bon compte, d'éblouissants effets de contraste peut-être, dans un langage nourri de sensualité, mais ne donnerait guère lieu à des jugements scientifiques sérieux et des conclusions durablement utiles. Plus nous sommes résolus à voir des biens culturels dans les créations d'un solide peintre, moins il est nécessaire de les classer dans les conditions particulières dans lesquelles elles se sont développées et s'affirment. Nous ne pouvons ignorer les points de vue d'une esthétique tournée vers la philosophie de la culture du XIX $x^{e}$ siècle, même là où nous n'en sommes encore qu'à nous assurer des conquêtes de notre mode d'observation purement artistique. La partialité formaliste de la comparaison exclusivement stylistique débouche sur une impasse et on comprendra suffisamment vite que cette impasse conduit ses maitres eux-mêmes jusqu'à l'absurde, attirant forcément sur des terrains mouvants les disciples qui leur emboîtent aveuglément le pas. Nos ancêtres heureusement "dépassés » de l'école hégélienne considéraient aussi toujours les arts particuliers sous l'angle de l'homme dans toute son étendue, de ce qui le concerne en général et de la tradition historique, un point de vue qui était devenu le leur parce qu'ils étaient les héritiers d'une époque florissante de notre philosophie et de notre littérature, ou bien parties prenantes de l'essor des études historiques ${ }^{18}$. Nous autres spécialistes modernes, nous n'avons besoin que de revenir à leur point de vue, ce dont nous nous sommes désaccoutumés, pour réaliser tant qu'il en est encore temps l'indigence de nos valeurs terriblement dégradées et sortir de notre myopie déjà très sensible. Fr. Th. Vischer, si décrié et pourtant toujours secrètement consulté, a résumé en ces termes la « véritable raison du classement » de l'ensemble de la peinture et de la sculpture «comme arts plastiques»: «le principe premier est ici dans le sujet, donc... dans le monde matériel initial ou bien dans les différences de l'imagination, la manière dont elle se porte ou se réfère à l'un ou l'autre de ces domaines matériels. C'est le caractère séparateur, exclusif du spatial, forme de base de tout art plastique, qui fonde cette classification : ce n'est pas la conformation de tel ou tel sujet qui est essentielle pour la différence entre les différentes branches artistiques, mais sa compréhension [Erfassung] » (\$696). Conformément à une époque où tout dépend de l'archéologie classique, il place au premier rang le tableau mythologique, car il représente toutes les ramifications [de la peinture] avant qu'elles ne se soient développées, ou bien les comprend déjà toutes en germe. La longévité de cet univers thématique reposait principalement sur le besoin constant d'une imagination tournée vers l'humain en général. Cependant: "Il faut en exclure le principal cercle de la foi chrétienne, qui est encore tenu de manière dogmatique et fait l'objet de controverses entravant pour la représentation toute chaleur humaine spontanée! » Pour l'art religieux, ou pour l'art religieux du Moyen Âge, il vaut mieux nous renseigner auprès du très profond H. G. Hotho qui fait précéder son « Histoire de la peinture chrétienne » restée inachevée du programme suivant: «Les problèmes à 
travers la richesse et la profondeur desquels entrent en jeu l'ensemble de l'imagination et toute exaltation de la forme et de la couleur, lui viennent d'abord de la vision chrétienne qui, en y apportant une solution heureuse, la porte à un point de vue d'où elle n'emprunte plus son style de présentation à d'autres arts, mais, faisant supérieurement valoir ce qu'elle est, transpose inversement par époques le proprement pictural, qui est son domaine, à l'architecture et à la sculpture ${ }^{19}$. " Puis, chez Vischer, vient ensuite la peinture de paysage du fait d'une "unité donnée des phénomènes de nature non organique et de nature végétale » avant qu'il n'en arrive, par le truchement $\mathrm{du}$ « tableau animalier », plus ou moins largement lié avec tel ou tel domaine voisin, au tableau de genre [Sittenbild] ${ }^{20}$ " qui appréhende l'homme d'un même point de vue que la peinture de paysage », c'est-à-dire où « l'homme apparaît malgré tout comme un être de nature au sens étroit et au sens large du terme - rattaché au général dans le sens du besoin, du travail, de l'état naturel et de l'état social, des formes de culture, bref de la coutume et des mœurs en général. » Le dernier champ thématique est celui de l'histoire, introduit par le portrait. La peinture appréhende ici l'humain en général dans la concrétion d'une action cruciale, gravée dans la mémoire de la postérité à travers un nom, un lieu et une date. L'autre fondement du classement est ici aussi «le sujet : d'abord la différence des temps, des peuples, des idées historiques ». La tentative de définir la différence entre le tableau de genre et le tableau d'histoire en parlant d'action pour l'un et d'état pour l'autre n'est, il est vrai, pas tenable, mais la solution ne peut se trouver que dans le degré différent d'évolution de l'objet de la représentation et ainsi de teneur de la culture intellectuelle. C'est sans doute aussi l'essentiel pour l'appréciation et le classement du paysage comme domaine de représentation propre de la peinture. Là où il apparaît comme développé, rabaissant les figures au rôle d'accessoires secondaires ou bien y renonçant tout à fait pour donner une portion de l'étendue de la nature pour elle-même, on peut dire de lui que « l'âme de la peinture de paysage, c'est le sentiment du monde. » C'est l'ensemble des relations avec le tout qui nous embrasse, et en lui, la signification de l'homme en tant qu'individu disparaît. Le pôle opposé de notre premier concept fondamental, «l'individu», est ainsi atteint: nous pourrions le désigner par le terme latin d'« universum». C'est entre les deux que s'accomplit toute la confrontation que nous considérons comme étant l'art. Cette sorte de peinture de paysage, Vischer, avec un net penchant au panthéisme, la définit en parlant de son caractère de " tableau d'atmosphère ", ou même « musical " ${ }^{21}$.

21 Sa classification ne se termine cependant pas sur cette division sommaire par sujets. Il se voit obligé d'appeler un second principe à la rescousse, néanmoins plus ou moins étroitement lié au principe de l'objet. Non seulement ce principe procède, comme chez plus d'un spécialiste de l'esthétique aujourd'hui encore, du domaine de la poésie - on ne s'en étonne d'ailleurs pas compte tenu de la formation d'abord littéraire de Vischer -, mais il se trouve également fondé, plus qu'il ne l'a entrevu, dans l'essence de la peinture même, art voisin de la poésie. Il faut simplement se garder de vouloir embrasser le domaine tout aussi hétérogène, peut-être même encore plus richement diversifié, de la représentation visible dans le cadre ordinaire des trois parties de la poésie. La même mise en garde vaut aussi ici vis-à-vis d'un usage direct des mêmes concepts fondamentaux, leur signification devant d'abord être modifiée avant de pouvoir être transposée. Car l'œuvre de peinture est muette et, liée à un instant, elle est de fait immobile, le mouvement n'y est qu'apparent, aussi suggestif qu'il soit pour l'imagination du spectateur. Cette dernière continue de composer au-delà de ce qui est donné, et le tableau visible n'offre d'abord que des valeurs mimiques, saisies et fixées 
dans leur apparition transitoire. Pourtant, ne serait-ce que parce qu'elle représente aussi l'environnement de l'unité de ce qui se passe entre des personnes et qu'elle restitue l'individu dans une situation, influencé par son milieu, la peinture dépasse l'ensemble des relations purement mimiques pour passer à des relations poétiques dont la spécificité particulière serait à définir, également par différence avec les relations picturales elles-mêmes. Les indéniables analogies, même sous réserve d'une terminologie strictement scientifique, ne peuvent s'expliquer de manière satisfaisante que si on les entend comme des correspondances entre l'organisation interne des champs artistiques et une activité de l'imagination qui n'a pas besoin de s'ouvrir au code logique de notre raison, parce qu'elle se trouve en réalité déjà fondée physiologiquement dans la psyché humaine ${ }^{22}$.

À ce stade, il faut retenir la correspondance manifeste entre la peinture et la littérature comme preuve du fait que toutes deux relèvent d'un degré supérieur de développement de l'esprit. C'est pourquoi il est important que les concordances observées aient plus d'une fois déjà conduit des penseurs avisés à admettre que l'art de la peinture, de la même famille, soit pénétré des mêmes principes que la poésie ${ }^{23}$. C'est ainsi que l'esthétique romantique expliquerait les choses, elle qui tend à accorder à la poésie, en tant qu'elle est l'art suprême, un rôle de guide de tous les autres, ou à la considérer, sinon comme l'origine, du moins comme l'âme de tout leur riche déploiement. Si l'on ne se laisse pas entraîner par la théorie à des prémisses métaphysiques, il faut souligner, à la suite de l'explication de Vischer, d'abord ceci. Si Vischer peut dire que «le spatial comme forme de base de tous les arts plastiques doit déterminer à travers son caractère séparateur, exclusif, également la classification de leurs champ particuliers ", avec la poésie, nous allons au-delà de ce spatial; avec sa forme de conception et de présentation, nous sommes déjà passés dans le temporel et sous l'emprise du déroulement ininterrompu du temps. Tout ce qui dans l'espace est "simultané » se dissout en une "succession » dans le temps, tout ce qui est matériellement coexistant se noie dans le flot des événements, de ce qui advient et passe. Les apparitions visibles s'évanouissent devant la trame de notre psyché qui les sous-tend toutes et dont les premiers mouvements les attachent au sentiment et à la volonté; ils sont transformés dans l'imagination, se condensent en idées qui s'inscrivent dans des concepts. Ce qui dans l'apparence colorée du tableau se transmet de la vie intérieure des hommes et des choses, ce qui, du monde intérieur, peut se révéler dans les relations visibles du monde extérieur, procède d'une autre nature que la nature spatio-corporelle des êtres et la commande avec tout ce qui peut y habiter. Nous touchons là à une énigme que nous ne pourrons élucider sans avoir auparavant exploré la spécificité de la poésie elle-même.

Restons-en donc à un exemple parmi les domaines de la peinture que nous avons nommés en dernier, peinture d'histoire ou tableau de genre. Nous avons de nouveau des personnes devant nous. Par le truchement des relations mimiques, nous sommes aussitôt conduits de l'ensemble des relations visibles à l'ensemble des relations invisibles, de l'extérieur corporel, avec des motifs qui frappent l'œil, à la communauté psychique, avec ses motifs intérieurs. L'itinéraire va donc directement de la reproduction peinte de la mimique, en tant qu'art expressif muet en soi, au contenu de la poésie qui, pour l'imagination du spectateur qui l'accueille, est inhérent à l'unité d'action des scènes de genre, du récit biblique ou légendaire, ou aux représentations 
dramatiques sans paroles, c'est-à-dire à la peinture silencieuse, comme si elles ne s'adressaient pas seulement au sujet, mais exprimaient objectivement ce contenu.

\section{Poésie}

Ainsi, nous sommes en mesure de déterminer la valeur spécifique que poursuit de son côté la littérature, qu'elle transmet et crée : c'est l'ensemble de relations motivées que les enfants des hommes que nous sommes, attendons, recherchons et organisons en tant qu'elles font agir notre volonté et constituent le moteur de nos actions. La motivation intérieure de tout événement et accomplissement est la valeur irremplaçable et indispensable que le poète, s'il veut rester un " poète », un « voyant " aux yeux des siens, saisit et représente, qu'il révèle à partir de sa connaissance de la nature humaine et de ses sentiments de la valeur, et qu'il doit lui-même inventer, compléter dans sa libre création même là où elle n'est pas saisissable, où elle n'existe peut-être absolument pas ou bien n'a pas laissé de trace identifiable. Faute de quoi, il en serait réduit à être un homme ordinaire comme les autres, ou à s'aliéner pour n'être qu'un historien consciencieux limitant ses propos aux objets de savoir ou de démonstrations à l'appui de preuves tandis qu'intérieurement il ne cesserait d'aspirer à ces valeurs suprêmes tant désirées qui ne veulent toujours pas se découvrir tout en étant enfouies, nous le croyons, comme un trésor que personne ne saurait voler. Et cette pénétration créatrice par l'imagination, avec la vie de notre vie intérieure, jusque dans les profondeurs cachées, ne s'arrête pas ici aux personnes humaines - avec lesquelles la mimique a exclusivement à voir FE⿱ $_{20}$, elle se porte aussi sur les relations avec les autres compagnons de notre existence, les animaux et les objets domestiques, les choses animées mais aussi inanimées de l'environnement. Elle s'étend aussi à toutes les parties constitutives du monde au dehors, jusqu'aux montagnes et aux fleuves sur le sol de la terre, aux nuages et aux étoiles dans le ciel. Tous les êtres et tous les phénomènes sont soumis au besoin qu'éprouve l'homme de trouver une explication de causalité [ursächliche Erklärung], ils entrent dans un ensemble de relations humainement compréhensibles, c'est-à-dire susceptibles de faire l'objet d'une expérience par le sentiment personnel. Et cette explication causale évolue également au cours du temps. L'explication personnificatrice selon la nature humaine, se voit peu à peu remplacée par l'idée de l'action de forces naturelles d'un autre ordre; mais tout en conservant l'analogie de l'acte de notre volonté et ainsi le noyau de l'action personnelle : ce n'est qu'avec la conception mécaniste que disparaît aussi le reste de la motivation poétique.

Saisissant ainsi avec audace les événements qui s'écoulent, l'imagination de l'homme s'approprie le monde entier, elle s'empare de l'instant fugitif pour en jouir, jusqu'au plus énigmatique, et en soustrait ainsi la valeur qu'elle attrape au vol pour en jouir encore et toujours; elle saisit le jeu de ces relations et de ces motifs, la totalité de l'explication du monde par des motifs extérieurs et intérieurs pour les faire resurgir comme une unité en perpétuel renouveau dans une langue formée d'un alignement de mots qu'elle assemble déjà en leur donnant ce sens en une phrase faite de sujets et de verbes. Ainsi, il est possible de réunir des masses importantes en une unité, de convoquer le chœur sur la scène dans la tragédie, et le peuple, l'armée entière dans l'épopée. Plus d'un concept abstrait pénètre ainsi dans l'imagination de celui qui écoute ou qui lit par le simple fait qu'il est désigné par un nom, un genre féminin ou masculin, comme une personne vivante, permettant ainsi au poète de maîtriser un collectif ou de 
rassembler une majorité dispersée de phénomènes, sinon séparés dans l'espace et dans le lieu!

Et comment définir ce moyen d'appréhension et de création de la valeur qui a été donné à l'homme, ou disons plutôt qui s'est développé dans la relation avec son semblable et le monde alentour, et que représente ce " $\operatorname{mot}^{24} »$ [Wort] ? C'est par ce terme que nous qualifions d'abord cette forme particulière que nos physiolinguistes qualifient de "geste sonore » [Lautgebärde], c'est-à-dire un complexe de voyelles et de consonnes (en réalité des éléments mimiques) ou inversement un geste de l'intérieur vers l'extérieur qui surgit, à l'aide du son de notre voix, du souffle d'air de notre respiration de sorte qu'il est d'abord audible, que le mouvement expressif jusque-là muet voire invisible (kryptomimique) est "prononcé » [verlautbaren, rendre sonore]. Mais nous appelons également Wort un assemblage de plusieurs mots de cette sorte, c'est-à-dire la phrase comme expression d'une unité de relations entre plusieurs mots, d'une idée aussi simple soit-elle. Le pluriel de ces phrases ne se dit plus Wörter [en allemand, le pluriel de Wort dans son sens de «mot»], mais Worte [en allemand, le pluriel de Wort prenant alors le sens de «paroles/verbe »]. Nous parlons de la parole du poète, du verbe de Dieu, du verbe de la sainte Bible [en allemand, différentes occurrences du mot Wort] ; c'est déjà une unité de composantes linguistiques qui fait du sens, une suite organisée de phrases qui ont un rapport intrinsèque entre elles, qui sont soumises à une idée supérieure, une idée dominante, et donc définissent un " ensemble de relations motivées ».

C'est pourquoi j'ai toujours comparé à dessein le Wort [mot/verbe] comme œuvre de la poésie au tableau comme œuvre de la peinture, en renvoyant au parallélisme de ces produits de l'esprit humain, ou d'abord de l'organisation humaine. Le tableau est l'unité visible des corps et de l'espace dans l'apparence de la surface, le mot est une unité audible de sons et de gestes, ou de mouvements sonores et de mouvements corporels, l'un étant un ensemble de relations optiques, l'autre de relations acoustiques. Mais le « tableau » n'est pas seulement l'œuvre visible du peintre du point de vue technique, en tant que portion bidimensionnelle d'espace et de corps sur la surface, cette définition vaut aussi pour toute portion aussi petite soit-elle d'une telle totalité, tant que les deux parties intégrantes de l'unité apparaissent d'une manière ou d'une autre, comme le montre très bien par exemple, pour la vision rapprochée, le fragment déchiré d'une gravure, d'une miniature ${ }^{25}$. Mais à quelle unité plus vaste renvoie en revanche le verbe dans [le verset] « Dis-le ce mot victorieux ${ }^{26}$ »! - mais de la même manière également le mot isolé, la succession de mots, de lignes du début jusqu'à la fin de la Bible que le copiste du Moyen Âge a dessinés avec tant de soin et de propreté, calligraphiés lettre après lettre, assemblés et dissociés mot après mot, alignés les uns après les autres. Cette brève mise en regard des deux formes d'expression de deux arts apparentés, la poésie et la peinture, est tellement importante et souhaitable parce qu'elle nous aide à envisager de plus près et à comprendre dans leur parenté la particularité des résultats à partir desquels nous les reconnaissons tous les deux. C'est aussi la principale intention des deux formules suivantes : $\sqrt{ }(\mathrm{R}+\mathrm{K})$ [Raum und Körper, espace + corps] pour le tableau et $V(\mathrm{~L}+\mathrm{G})=$ son et geste [Laut und Gebärde] ou ton et geste [Ton und Gebärde] (voyelle et consonne), donc $\checkmark(T+G)$ pour le mot, dans lequel l'élément du ton se combine avec la gestuelle, le mouvement expressif, et fait ainsi surgir l'ensemble des relations naturelles avec la musique et la mimique. Les deux formules illustrent l'origine des produits en question, comme résultats d'une réduction des valeurs pleines et entières sur lesquelles ils reposent qui, en renonçant à une partie de la force sensible 
des deux parties fondues ensemble atteignent à une synthèse supérieure pour plus d'énergie intellectuelle. La rapidité du mot fougueux va au-delà de la mobilité de la gestuelle corporelle, du jeu de la physionomie, par la combinaison des gestes retenus ou se déroulant seulement de manière crypto mimique et du ton de la voix, tandis que le son (Laut), en renonçant aussi à la force pleine de l'expiration, combiné à l'expression mimique atteint à l'objectivité de qualifications concrètes qui reste interdite au ton (Ton) dans sa vibration librement évanescente. Mais le tableau ne peut aussi embrasser les choses qu'en sacrifiant la troisième dimension des choses réelles, celle de l'espace autour, et, avec la même préoccupation intellectuelle, se détache de l'impression visible de la réalité en trois dimensions pour atteindre à une synthèse plus élevée. La définition de leurs éléments fondamentaux, le tableau comme le mot, suffisent à constater que les deux arts reposent sur des résultats psychiques plus combinatoires, donc plus avancés, sur des conquêtes indéniablement plus libres de la vie intellectuelle que les autres arts dont nous pouvons comparer les éléments fondamentaux, c'est-àdire le corps pour la sculpture, l'espace pour l'architecture, ou le son pour la musique et le geste (ou mouvement corporel comme expression) pour la mimique. C'est pourquoi justement ces deux derniers arts particuliers, la peinture et la poésie, doivent occuper aux yeux du théoricien comme du psychologue un rang supérieur et, du fait de leur parenté intrinsèque, être compris comme un couple. C'est seulement à travers eux que la confrontation de l'homme avec le monde conduit à une unité artistique de l'intuition du monde; ces deux arts sont issus du "sentiment du monde » et, déterminés par lui, sont en quête des valeurs que leur œuvre nous représente et que, ce sentiment, libéré de la pesanteur du monde matériel, transmet à la libre imagination, au besoin de création de la forme d'expression.

Nous entendons que, comme pour la peinture, soit établi pour la poésie un concept général qui soit l'« idéal-type » de cet art; c'est pour cet art qu'il semble le plus aisé à comprendre que nous utilisons continuellement une sorte d'image mentale permettant d'aborder la littérature de tous les temps et de toutes les époques et d'en dégager le critère permettant de définir ce qui, en elle, peut être reconnu comme poésie, comme art de la poésie. Tout à fait naturellement, nous accordons à ce concept idéal une valeur normative. Mais dans l'application à un «individu historique " (ici au sens de la philosophie de l'histoire ou des sciences de la culture) s'ensuit immédiatement une subdivision de cette unité abstraite en genres que nous appelons volontiers les genres épique, lyrique (poétique) et dramatique. Ils sont hérités de l'observation historique et de l'observation théorique de la poésie et nous avons vu Fr. Th. Vischer les utiliser comme des catégories courantes dans son esthétique, les reprendre tout simplement du domaine de la peinture. De notre point de vue, il faudrait certainement toujours rendre compte d'une « transposition » de concepts élaborés sur un autre terrain, et apporter la preuve de leur justification pour le royaume de la poésie. Un examen de fond porterait aussi peu tort à notre actuelle esthétique de la poésie qu'à l'histoire de la littérature et proposerait certainement des conclusions bienvenues à l'exploration psychogénétique de l'imagination. Quoi qu'il en soit, il y a dans cette articulation en trois parties, même si les dénominations n'en sont pas une expression parfaite, une tentative d'établir un lien entre le contenu et la forme, de la même manière que se fait chez Vischer la transition entre la répartition des « sujets » et des « moyens artistiques ». Et la question principale serait de savoir jusqu'à quel point ici le contenu et la forme peuvent se recouvrir, jusqu'à quel point donc des points de vue de philosophie de la culture peuvent coïncider avec des points de vue de la science de l'art ou de l'esthétique. L'une 
des grandes erreurs dans cette sorte de considérations réside à mes yeux dans le fait qu'on ait négligé la mimique comme art indépendant et dans le péché par omission qui en découle, à savoir le fait qu'on n'ait pas salué comme elle le mériterait la part prise par le caractère «mimique » dans les arts voisins, sa présence donc dans la musique d'abord, puis sous une forme intellectualisée également dans la poésie. Nous sommes ainsi de nouveau confrontés à la tâche que nous avons définie auparavant sans vouloir $\mathrm{y}$ apporter une solution, mais il n'est plus besoin ici de continuer plus avant et encore moins de donner une réponse ultime, parce qu'il ne s'agit, dans tout ce programme, que de désigner les problèmes et de les envisager de notre double point de vue. Dans le chemin que nous avons parcouru, la conclusion est apparue d'elle-même que seul le recours aux idées de valeur semble apte à dégager une communauté satisfaisante de concepts fondamentaux et ainsi d'envisager une synthèse supérieure entre science de l'art et philosophie de la culture. Ainsi, grâce à une théorie des valeurs accomplie, reposant sur des fondements tout à fait concrets, nous arriverions à un "système de valeurs " général qui devrait comporter en soi une hiérarchie. Si l'on me demande encore comment je pense l'établir, je renverrai volontiers aux développements très approfondis de Max Scheler, qui me sont personnellement sympathiques, tels qu'ils ont été formulés en détail en 1915 (Scheler 1913-1916) ${ }^{27}$ dans son traité «Le formalisme dans l'éthique et l'éthique matérielle des valeurs (en particulier de l'éthique d'Emmanuel Kant) »[Der Formalismus in der Ethik und die materiale Wertethik (mit besonderer Berücksichtigung der Ethik Immanuel Kants)].

\section{Généalogie des arts}

Pour pouvoir les ranger dans ce système de valeurs, la science de l'art doit nécessairement commencer par envisager la généalogie des arts que nous venons de fonder dans le parallélisme entre la peinture et la poésie, ainsi que leur prééminence par rapport aux couples plus originaires que sont la sculpture et la mimique, l'architecture et la musique; de même, il ne devrait pas y avoir de doute quant à la relation d'équivalence entre les deux termes du premier de ces couples malgré toute l'opposition entre la forme de vision spatiale d'un côté et la succession temporelle de l'autre. Si d'un côté la peinture apparaît comme une union supérieure de ce que traitaient et visaient avant elle la sculpture et l'architecture, les valeurs d'existence que sont le corps et l'espace, de l'autre côté la poésie est l'union supérieure de ce que traitaient et visaient la mimique et la musique, les valeurs de vie que sont la gestuelle et la voix, ou le mouvement corporel et le mouvement sonore comme expression du monde intérieur. Dans ces stades antérieurs, la mimique et la sculpture vont indéniablement de pair en tant qu'entité double où l'une exige et déclenche l'autre, comme dans un jeu d'échange fondamental entre deux partenaires inséparables, fondateurs de «toute forme d'expression " créatrice venant de l'homme et destinée à l'homme. De la même manière, la musique et l'architecture, bien que l'une s'inscrive dans le temps et l'autre dans l'espace, apparaissent comme des conquêtes de la même famille, dépassant l'homme, conquête de l'espace pour la seconde, du temps pour la première, s'accomplissant dans le monde intérieur d'un côté, dans le monde extérieur de l'autre. Toutes deux procèdent des lois de l'organisation humaine pour atteindre à une forme plus vaste d'expression et à la création de nouveaux univers déterminés par les lois de l'art. Cette relation est également confirmée par les articulations de transition ou les royaumes intermédiaires situés entre les deux derniers couples d'arts 
particuliers autonomes : entre la mimique et la musique - la danse, comme mouvement expressif du corps humain en rythme 医, et entre la sculpture et l'architecture - la tectonique, en tant qu'elle forme des corps non organiques et constitue un stade préliminaire de l'architecture 漪. Nous laisserons d'abord de côté la manière dont se réalise le parallélisme entre ce dernier couple des arts particuliers; car dans la première réponse apportée à la question des concepts fondamentaux communs à la science de l'art et à la philosophie de la culture, il s'agit plutôt de nous limiter aux arts plastiques. L'architecture et la musique demanderaient à être fondées par la psychophysique et une synthèse psychogénétique, pour que puissent se dégager pour elles aussi des conclusions concises et compréhensibles. Toutes deux demanderaient surtout que l'on revienne aux débuts de l'art dans la vie culturelle des peuples, ce qui par rapport au propos qui est le mien ici, nous mènerait trop loin et trop en dehors de mon sujet ${ }^{28}$. Il va néanmoins de soi que précisément ce retour aux temps préhistoriques ou à la situation des peuples primitifs doit faire ressortir toute la série de points de vue alors courants de l'histoire de la culture et des sciences sociales. En ce qui concerne l'architecture en particulier, si l'on s'interroge sur les raisons extérieures et les impulsions intérieures qui présidèrent à leur création, on voit surgir les concepts fondamentaux des phénomènes culturels qui expliquent comment les hommes, pour vivre ensemble, se réunissent en clan et famille, en tribu et en horde, en cité et autres formes d'organisation destinées à les protéger. De la tente à la hutte, de la maison au temple, du village à la ville, la progression se fait vers une mise en architecture de l'unité politique ou religieuse, de l'incarnation spatiale de la communauté des corps jusqu'à celle d'une communauté des esprits, comme le cours de l'histoire accompagne et fait avancer l'architecture " configuratrice d'espace ». L'« idéal-type » de l'État ou de l'Église, des mœurs ou de la loi etc., comme dans les sciences de la culture, intervient ici aussi. L'opposition entre volonté individuelle et volonté sociale nous invite nous aussi à regarder du côté des raisons psychologiques. Quelle opposition y a-t-il par exemple déjà entre le temple antique, comme lieu de l'individu divin sous la forme d'une statue, et la basilique chrétienne comme espace de rassemblement de la communauté ou comme espace d'action de la communauté au-dessus de l'individu dans sa relation avec le Dieu invisible ${ }^{29}$.

30 Pour la musique, nous pouvons dire ici à quel point un lien avec les travaux de Wallaschek et Stumpf serait souhaitable, tandis que d'un autre côté naturellement il faudrait également d'urgence élaborer des concepts pour une théorie et une histoire des stades d'évolution supérieurs, comme c'est le cas par exemple dans le panorama historique et critique de Paul Moos sur l'esthétique musicale allemande de Kant jusqu'au milieu du XIX $x^{e}$ siècle, et de là jusqu'à nos jours, sur la base de la théorie d'Eduard von Hartmann. Mais, selon moi, une autre exigence découle du fait que la mimique naturelle s'est avérée le lieu d'origine de toute forme d'expression et de l'art éponyme qui constitue le chaînon intermédiaire entre la musique et la poésie. Comme dans tout acte et tout geste créateur de notre corps humain, les mouvements corporels jusqu'aux jeux de notre physionomie, en tant qu'expression des mouvements affectifs et des mouvements de l'âme en général, vont de l'intérieur à l'extérieur, de la m ême manière les mouvements du son de la voix humaine, jusque dans ses plus délicates vibrations, sont fondés sur la même activité d'expression. Et la production de sons par des instruments artificiels, la répartition des différentes voix sur ces derniers qui, à l'origine, ne devaient servir qu'à accompagner le chant pour relayer l'organe humain, toute la tendance à l'autonomisation et le perfectionnement qui s'y manifestent se font 
cependant toujours par analogie avec le mouvement immédiat que l'homme exprime par sa propre voix. C'est de cet ensemble de relations génétiques que se développe toute la rythmique, celle des mouvements corporels comme celle des instruments, et il est fatal de voir dans le monde des sons un monde seulement acoustique distinct de ce rythme, et son art comme un jeu complètement libre avec de purs éléments sonores, suspendus quelque part dans les airs. Toute la conquête du "temps " pour réaliser et faire vivre un art humain à partir de tels symboles expressifs n'est pensable et réussie que sur le fondement du mouvement propre de la voix et du corps de l'homme, et donc finalement sur le fondement du même support que celui auquel la mimique doit ellemême aussi son origine et en même temps son essence. À partir de là, l'on peut donc aussi conclure à un concept fondamental propre à définir l'essence de la musique en tant qu'art. L'« idéal-type » que nous formons doit saisir la valeur qu'elle poursuit dans sa quête pour la transmettre et la mettre en forme pour qu'elle soit un objet toujours renouvelé d'expérience vécue. C'est la valeur la plus volatile de toutes, une valeur de la vie de l'âme, le sentiment, le «mouvement de l'âme » qui frémit et palpite en nous.

Ce n'est cependant pas le perfectionnement de la théorie de la musique - que nous espérons de l'étude de l'image mimique première de tout jeu sonore -, que nous envisageons ici, mais l'aspect général de l'ensemble des relations de tous les arts avec l'organisation humaine qui est le seul à pouvoir dégager une perspective sur toute l'étendue des exigences de la philosophie de la culture. Quand on y songe, il faut alors admettre cette exigence de concepts fondamentaux communs pour la science de l'art également. Il y a là tout un grand chapitre que les représentants de la théorie de la musique ont d'autant plus longtemps négligé qu'eux-mêmes n'étaient pas suffisamment en contact avec le reste des sciences de l'esprit, justement dans le sens de la culture. Une phénoménologie de la musique avec toutes ses formes historiques et aujourd'hui encore présentes a besoin des clés de la psychologie, et il lui faut se concevoir comme une science de la culture pour amasser encore les fruits mûrs d'une récolte tardive.

Mais partout se fera également sentir la nécessité de rigoureusement distinguer d'abord la musique pure de tous les liens qu'elle entretient avec les arts voisins, que ce soit dans le chant avec l'art des mots, ou dans le drame musical avec la mimique, mais aussi avec le théâtre et la poésie, et donc la nécessité de la traiter séparément. Il faudra alors, avec toute la discipline scientifique requise, sanctionner imperturbablement la confusion terminologique et le démon de l'analogie, faute de quoi il ne sera pas possible de faire toute la clarté et de rendre autant compte des phénomènes purement artistiques que de leur ultime signification sur le plan de la philosophie de la culture. Là aussi, c'est seulement grâce aux idées de valeur et aux idéal-types qui leur correspondent que l'on éclairera complètement les sentiers tortueux de l'évolution historique.

Notre tentative de suivre la trace du sentiment de valeur dans la création de chacun des arts en particulier, et de rapporter les réponses aux idées de valeur que cette tentative a pu suggérer au point de vue de la philosophie de la culture nous permet de reprendre la division en domaines de représentation par le contenu, longtemps ajournée et oubliée, voire même méprisée, sous le coup des querelles esthétiques, et de la recommander à une refondation des riches conclusions que nous a léguées l'heure de gloire de notre philosophie. Si ce sont vraiment les « valeurs d'existence et de vie » qui fondent les limites et les articulations de ce système, elles sont pleinement justifiées ; 
elles vont s'avérer fécondes, pour nous encore. Et si le pur ressort de la création, c'est le sentiment de valeur avec son amour qui vient progressivement à l'homme au cours de sa maturation affective [seelisch] et intellectuelle, ou qui lui ouvre de nouvelles perspectives inespérées à certains tournants de cette évolution, qui soulève son enthousiasme en lui faisant franchir un pas nouveau vers le royaume des idéaux - alors nous pouvons nous attendre à ce que se révèlent des territoires insoupçonnés et que soient récoltés les fruits de nouveaux élans que toute saine philosophie de la culture reconnaîtra avec joie.

Si nous reprenons encore une fois pour finir les deux arts dont l'un fait partie de l'hémisphère du temps, l'autre de l'hémisphère de l'espace et qui forment un couple complémentaire pour une forme d'expression cohérente dans son unité, parfaite dans son genre, nous voyons qu'ils proposent ensemble une vision artistique du monde ou une résurrection du monde d'un caractère particulier auquel correspond le caractère des grandes époques de culture. À l'intérieur de ces possibilités objectives de l'évolution, il ne s'agit que de savoir auquel de ces deux arts revient l'hégémonie que subissent aussi les autres arts, et quand, dans le mouvement de pendule entre les deux, la prédominance passera à l'autre art complémentaire qui fera valoir le courant opposé. Ce n'est aucunement un qualificatif abscons, qui irait chercher "pour faire de l'esprit " l'idée la plus éloignée, que de désigner comme je l'ai fait ces couples d'arts qui se complètent, qui s'appellent intrinsèquement et se relaient effectivement l'un l'autre, par la formule d' «arts complémentaires ». Le premier de ces couples est le couple que forment la mimique et la sculpture. Longtemps, ils furent les seuls à assumer, avec alternativement l'un ou l'autre plus de poids, la confrontation artistique de l'homme avec le monde. C'est l'époque primitive de la causalité personnaliste qui est indubitablement dans ce lien. Mais c'est aussi, sous l'hégémonie de la sculpture, la condition préalable de l'Antiquité classique. Le deuxième couple est celui que forment la poésie et l'architecture. Si tous les deux agissent et portent leurs fruits en se complétant, la vision du monde d'une époque n'a pas besoin, pour son expression artistique, de la participation d'autres arts qui, de toutes façons, subissent leur influence déterminante. L'exemple le plus frappant en est l'art de la totalité du Moyen Âge, la poésie en dominant la première moitié - avec naturellement les écritures saintes et l'ensemble de la doctrine de l'Église chrétienne-, tandis que la seconde période, la période gothique, connaît l'exclusive hégémonie de l'architecture. Reste le troisième couple de cette sorte, celui de la musique et de la peinture qui semblent tellement éloignés que supposer entre eux une interaction ne peut que susciter l'étonnement et le doute. Mais nous reconnaissons depuis longtemps déjà en eux les arts spécifiquement modernes qui distinguent la culture récente par leurs succès et par la préférence qui leur est accordée. Et ces deux arts ne confèrent-ils pas à ces périodes de l'évolution de l'art un caractère esthétique spécifique? - et l'image du monde qu'ils nous livrent ne comporte-t-elle pas d'autres couleurs ou d'autres nuances? Tout a commencé par l'essor de la peinture. Nous savons exactement depuis quand cet essor s'est transformé en triomphe qui a imposé aux autres arts la "vision picturale ». L'art de la poésie n'a-t-il pas fini par peindre lui aussi ? Une éclosion inédite de la musique a suivi, relayant l'autre art pour donner le ton. Puis de nouveau, après le travail de préparation des courants souterrains à l'œuvre dans les autres arts, la réforme de la poésie et le retour à une architecture sobre et ainsi un nouveau retour de balancier à la fin du XVIII ${ }^{\mathrm{e}}$ siècle. Et ensuite, au XIX ${ }^{\mathrm{e}}$ siècle ? Une indéniable prédominance de toute la littérature à la suite de la supériorité classique; la peinture, retenue depuis 
Winckelmann dans l'imitation de la sculpture antique, s'efforçant tant bien que mal depuis la réaction romantique de se concentrer sur sa propre identité, tenue à de longues années d'apprentissage, pénétrée de poésie dans sa forme graphique, régentée même par cette dernière dans l'art monumental. La musique elle-même, animée du plus fervent besoin de s'allier au verbe, et (à travers Wagner) exaltée jusqu'à devenir " une œuvre d'art totale ». Au terme de tout cela, l'avenir n'appartient-il pas au Xx siècle à l'art le plus proche dans la famille des arts, à l'architecture qui désormais, après avoir repris tous les styles avec une application scolaire, après avoir recommencé depuis le début avec les arts appliqués, a recommencé (enfin, enfin! disons-nous, nous autres contemporains dont le regard est si myope) à se déployer par elle-même dans sa liberté créatrice - comme le voulait son destin pour qu'elle soit "configuratrice d'espace » en harmonie avec son essence la plus propre ${ }^{30}$.

Comment se fait tout cela? demandons-nous. Chacun des arts en particulier, créant en accord avec lui-même, acquiert nécessairement une perfection jusqu'à un certain degré toujours plus grande qui influence les arts voisins. Mais la pérennité de cet effet nécessite que soit compensée cette dépense d'énergie ; parce qu'aucune force ne jaillit d'un organisme vivant sans qu'il ne s'épuise et que ce renouvellement permanent ne finisse par se tarir, la supériorité d'une source d'énergie se déplace vers une autre, d'un pôle de tension vers le pôle opposé. Et les modalités de passage d'une cellule noyau à une autre sont nombreuses : ou bien il y a une mutation à bas bruit, imperceptible, de proche en proche, dans les arts les plus voisins ou bien c'est un changement rapide, dans un conflit brutal entre deux antipodes qui se complètent néanmoins et se relaient et dont je ne saurais nommer la nature profonde plus simplement qu'en les qualifiant comme les couleurs que le physicien appelle « complémentaires ». Que nous désignions l'idéal-type d'un art dans le sens du biologiste par le terme d'être vivant psychophysique, ou dans le sens du spécialiste de la culture par le terme d'«individu historique ", notre besoin de causalité revient malgré tout toujours dans le premier cas peut-être davantage à une interprétation dynamique de la cause, dans le second en revanche à une interprétation personnaliste. Le principal reste de parvenir en réunissant deux arts qui se complètent et travaillent ensemble, en tant que couple déterminant, à une forme d'expression unique d'une vision du monde momentanée, à toute une série exceptionnellement instructive et féconde d'idéal-types ou de caractères artistiques supérieurs de cultures tout à fait différentes auxquels correspondent, dans l'évolution historique, de véritables "individus historiques et actuels » dans le sens de la sociologie ou de la philosophie de la culture. Ce sont les ultimes concepts fondamentaux que la science de l'art, j'en suis convaincu, peut offrir dès maintenant. Ils pourraient selon moi servir encore mieux la philosophie de la culture que ne l'a fait récemment encore le livre de Mehlis qui ignore tout le travail de l'histoire de l'art ou ne le prend pas en considération comme il le devrait. 


\section{BIBLIOGRAPHIE}

Fouillée A. (1890) : L'évolutionisme des idées-forces, Paris, Alcan.

Fouillée A. (1908 [1890]) : Der Evolutionismus der Kraft-Ideen, traduit vers l'allemand par R. Eisler, Leipzig, Klinkhardt.

Heyne M. (1890-1895) : Deutsches Wörterbuch, Leipzig, Hirzel, 3 vol.

Heyne M. (1890-1895) : Deutsches Wörterbuch, Leipzig, Hirzel, 3 vol.

Hotho H. G. (1835) : Vorstudien für Leben und Kunst [Préliminaires pour la vie et l'art], Stuttgart et Tübingen, Cotta.

Lask E. (1902 [rééd. 1914]): Fichtes Idealismus und die Geschichte [L'idéalisme de Fichte et l'histoire], Tübingen / Tübingen, J. C. B. Mohr.

Meumann E. (1908) : Einführung in die Ästhetik der Gegenwart [Introduction à l'esthétique du temps présent], Leipzig, Quelle \& Meyer.

Ritschl O. (1901) : Die Kausalbetrachtung in den Geisteswissenschaften [La causalité dans les sciences humaines], Bonn, Georg.

Scheler M. (1913) : Zur Phänomenologie und Theorie der Sympathiegefühle, von Liebe und Hass [De la phénoménologie et de la théorie des sentiments de sympathie, d'amour et de haine], Halle, Niemeyer.

Scheler M. (1913-1916) : « Der Formalismus in der Ethik und die materiale Wertethik (mit besonderer Berücksichtigung der Ethik Immanuel Kants) » [Le formalisme en éthique et l'éthique matérielle des valeurs], 1 ère et $2^{\text {ème }}$ parties, dans Husserl E. (éd.) : Jahrbuch für Philosophie und phänomenologische Forschung, Halle, Niemeyer, vol. 1, p. 405-565 et vol. 2, p. 21-478.

Schmarsow A. (1905) : Grundbegriffe der Kunstwissenschaft am Übergang vom Altertum zum Mittelalter [Concepts fondamentaux de la science de l'art], Leipzig, B. G. Teubner.

Tarde G. (1899) : Les lois sociales : esquisse d'une sociologie, Paris, Alcan.

Uexküll J. von (1909) : Umwelt und Innenwelt der Tiere [Milieu et monde intérieur des animaux], Berlin, Springer.

Uexküll J. von (1913) : Bausteine zu einer biologischen Weltanschauung [Éléments d'une vision biologique du monde] Munich, Bruckmann.

\section{NOTES}

1. Littéralement, en allemand : image debout, dressée. NDT.

2. Lask (1902 [rééd. 1914]), p. 11 et suiv. Certaines références bibliographiques données par August Schmarsow étant trop lacunaires, la traduction n'a pas pu les restituer. Par ailleurs, sauf indication bibliographique, les traductions sont de la traductrice. NDT

3. Littéralement, en allemand : image-colonne. NDT

4. Cf. mes « Concepts fondamentaux de la science de l'art dans la transition entre l'Antiquité et le Moyen Âge » [Grundbegriffe der Kunstwissenschaft am Übergang zwischen Altertum und Mittelalter], 1905 , ch. XII. 
5. L'auteur utilise dorénavant presque systématiquement l'équivalence qu'il a établie plus haut entre la notion de «sculpture » et celle de «formatrice de corps » [Körperbildnerin]. NDT

6. On peut également parler de "figures engainées ", la gaine étant le socle se poursuivant insensiblement, sans rupture, par une figure en ronde bosse de tête ou de mi-corps. NDT.

7. Cf. Heyne (1890-1895).

8. La distinction s'opère en allemand entre le corps strictement physique et matériel, le corps dans l'espace sans conscience [Körper] et le corps qui a conscience d'être un corps [Leib]. NDT.

9. Cf. Uexküll (1913), (1909). - Scheler (1913), (1913-1916).

10. Ritschl (1901).

11. Cf. Ritschl, ibid., qui propose une histoire convaincante de l'évolution du concept de causalité dans ses grandes lignes. Cette imputation a été aussi admise par Max Weber comme racine de la causalité

12. Nous trouvons une extension récente de l'usage dans le domaine de la philosophie de l'histoire. Quand nous pensons à un "individu historique ", nous pensons quant à nous à un personnage historique. Les sciences de la culture entendent quant à elles sous cette dénomination des complexes entiers de phénomènes historiques dans leur conditionnalité spécifique, pour autant que leur cohérence interne et externe soient propres à être considérées comme une unité. « Nous disons maintenant individu historique », écrit Max Weber, « rejoignant ainsi une expression parfois utilisée par la méthodologie de la science sociale et qui est devenue courante dans la logique à travers une formulation précise, pour signifier un phénomène culturel. » Dans la science de l'art, nous préférons limiter l'usage à des individus concrets.

13. «Le contact d'un esprit avec un autre esprit est, en effet, du point de vue de chacun d'eux, un événement tout à fait à part, qui se détache vivement de l'ensemble de leurs contacts avec le reste de l'univers et donne lieu à des états d'âme des plus imprévus, des plus inexpliqués par la psychologie physiologique.", Tarde (1899) p. 28 ; «...[Je prétends que] le rapport de ces deux personnes [un couple de deux personnes à quelque sexe qu'elles appartiennent] est l'élément unique et nécessaire de la vie sociale [et qu'il consiste toujours, originairement en une imitation de l'une part l'autre]. ", ibid, p. 35-36.

14. L'ensemble du raisonnement repose sur le champ lexical formé autour de Schau-, de schauen : voir et/ou donner à voir. L'acteur, Schau-spieler est celui qui joue à donner à voir, et le spectacle ou théâtre est le Schau-spiel, le jeu qui donne à voir. D'où la comparaison avec la mimique, qui ne donne pas à entendre... NDT.

15. Je ne comprends vraiment pas comment j'ai pu être si souvent mal compris, quand on me reprochait de ne voir dans la mimique rien d'autre que l'art du théâtre. Ce reproche d'étourderie dans le jugement touche également un ancien auditeur. Meumann (1908), p. 129.

16. L'étude de la peinture en tant qu'art de la surface, c'est-à-dire son aspect décoratif ne seront volontairement pas traités ici.

17. Scheler (1913-1916), vol. 2, p. 172.

18. Permettons ici de rappeler la parallèle avec le couple développé plus haut que constituent l'individu et l'individualisme ; lui correspondrait ici - étant donné le sentiment du monde dans la peinture et la reconnaissance des relations déjà préparées par la mimique - le couple communauté et collectivisme ou même universalisme.

19. Cf. également ses cours sur « L'école de peinture d'Hubert van Eyck avec ses prédécesseurs et contemporains allemands[Die Malerschule Huberts van Eyck nebst deutschen Vorgängern und Zeitgenossen], Berlin, 1855 et 1858, ainsi que Hotho (1835) dont le point de départ est le Don Juan de Mozart.

20. Littéralement, un Sittenbild est un tableau de mœurs, le terme comportant donc une connotation morale, mais cette catégorie n'existant pas vraiment, il s'agit sans doute ici de ce qu'on appelle le tableau de genre. NDT. 
21. Quand Vischer ne voit dans le paysage que le domaine de la poésie, du tableau d'atmosphère, c'est un jugement tout à fait étroit et qui ne correspond qu'à la manière de voir du XIX ${ }^{e}$ siècle. Là aussi, on voit se manifester la métamorphose de la culture intellectuelle qui conditionne aussi la métamorphose de la culture esthétique.

22. Voir sur ce point Wilhelm Ostwald, «Energetische Grundlagen der Kulturwissenschaft » [Fondements énergétiques de la science de la culture], in : Philosophisch-soziologische Bücherei, vol. XVI, Leipzig 1909, et Alfred Fouillée, « Évolutionisme des idées-forces » (traduit en allemand par Eisler en 1908).

23. Voir sur ce point mes explications au sujet du Laocoon de Lessing, Leipzig, 1907.

24. Le même terme de Wort exprime en allemand le mot (au singulier) en tant qu'entité linguistique, le verbe, notamment au sens biblique, la parole (la capacité de parler, et ce qui est dit). Nous garderons le terme allemand de Wort en indiquant sa valeur en français entre crochets. NDT

25. Il suffit de penser au jeu dans lequel une image a été collée sur une plaque de bois découpée de manière irrégulière en plusieurs morceaux, et qui consiste à assembler de nouveau ces morceaux aux contours sinueux et arbitraires pour reconstituer l'image [puzzle].

26. Il s'agit d'un verset du psaume 46, dans le texte de Martin Luther : Das Wort, sie müssen lassen stahn, extrait de Eine feste Burg ist unser Gott; Deus noster refugium et virtus. Il existe dans deux traductions françaises que nous indiquons ici: 1) «Il suffit d'un mot de Dieu/Dis-le ce mot victorieux» (Henri Lutteroth, 1847) 2) «Un seul mot, ô chrétien, terrasse l'adversaire/Le mot c'est du grand Roi des rois la Parole immortelle » (Recueil de Montbéliard, 1847). NDT.

27. La date donnée par A. Schmarsow n'est pas correcte. Ce texte de Scheler a été publié en deux articles séparés, le premier en 1913, le second en 1916. NDR.

28. Comme il n'est pas encore possible de reconnaître l'ornement comme un art indépendant parce qu'il ne représente lui-même aucune valeur, mais ne fait qu'en souligner et accompagner certaines, préexistantes, comme donc je le considère comme un stade antérieur à l'art, j'ai traité dans cette revue (vol. 5 (2 et 3$)$ ) des "Raisons premières de l'ornement " [Anfangsgründe der Ornamentik] en ce sens que les fondements communs que l'on y trouve peuvent être transposés aux autres arts musicaux et littéraires [musisch, pour les trois Muses originaires de la musique et de la littérature]; et je propose ici expressément d'étudier le rôle et l'importance de la part de l'ornement dans la musique, la danse, dans la mimique et enfin dans l'art du mot.

29. Je renvoie ici à mes «Principes fondamentaux de la science de l'art dans la transition entre l'Antiquité et le Moyen Âge » [Grundbegriffe der Kunstwissenschaft am Übergang zwischen Altertum und Mittelalter] (Schmarsow 1905), où je n'ai cessé de me préoccuper d'un terrain d'entente avec les sciences de la culture autant que le permettait une discussion avec des points de vue divergents.

30. Cf. ma leçon inaugurale de Leipzig sur l'«Essence de la création architecturale ", le 8 novembre 1893. 
INDEX

Mots-clés : philosophie de la culture, généalogie des arts, valeur artistique, corps/corps propre, individu, idéal-type

Schlüsselwörter : Kulturphilosophie, Genealogie der Künste, Wert der Kunst, Körper/Leib, Einzelwesen, Idealtypus

\section{AUTEURS}

AUGUST SCHMARSOW

Historien de l'art (Plus d'informations ici) 